\title{
A FINITE FORM FOR THE WRAPPED POISSON DISTRIBUTION
}

\author{
FR.ANK BALL, ${ }^{*}$ University of Nottingham \\ PAUL BLACKWELL, ${ }^{* *}$ University of Sheffield
}

\begin{abstract}
We give a finite form for the probability mass function of the wrapped Poisson distribution, together with a probabilistic proof. We also describe briefly its connection with existing results.
\end{abstract}

AMS 1991 SUBJECT CLASSIFICATION: PRIMARY 60E99

SECONDARY 60J27; 33B99

If $N$ has a Poisson distribution, then we say that $M=N \bmod k$ has a wrapped Poisson distribution on $\{0,1, \cdots, k-1\}$. Lévy (1939) and Mardia (1972) use an equivalent form, the distribution of $(N / k) \bmod 1$ on $\{0,1 / k, \cdots,(k-1) / k\}$.

The probability mass function for the wrapped Poisson distribution can readily be written in terms of infinite sums. The main aim of the current paper is to express these probabilities in a finite form, which appears to be previously unpublished (Blackwell (1990)). We indicate two approaches to the proof of this result.

Theorem. Let $N$ have the Poisson $(\lambda)$ distribution. The corresponding wrapped Poisson distribution on $\{0,1, \cdots, k-1\}$ has probabilities given by

$$
\begin{aligned}
& \operatorname{Pr}(N \bmod k=n) \\
& =\frac{1}{k} \exp (-\lambda) \sum_{j=0}^{k-1} \exp \left(\omega_{k}^{j} \lambda\right) \omega_{k}^{-n j} \\
& =\frac{1}{k} \exp (-\lambda) \sum_{j=0}^{k-1} \exp (\lambda \cos (\theta j)) \cos (\theta n j+\lambda \sin (\theta j)) \quad(n=0, \cdots, k-1),
\end{aligned}
$$

where $\theta=2 \pi / k$, and $\omega_{k}=\cos \theta+i \sin \theta$ is a complex $k$ th root of unity.

Proof. Using the fact that the quantities in the theorem are probabilities, and hence real, the complex expression (1) can readily be rewritten in the form (2), involving only real functions. Therefore it is sufficient to prove (1).

One approach to the proof of (1) is via the $k$ th-order hyperbolic functions, given by

$$
J_{k}^{n}(\lambda)=\sum_{m=0}^{\infty} \frac{\lambda^{n+m k}}{(n+m k) !} \quad(k=1,2, \cdots ; n=0, \cdots, k-1) .
$$

Received 7 May 1991; revision received 27 August 1991.

* Postal address: Department of Mathematics, University of Nottingham, University Park, Nottingham NG7 2RD, UK.

${ }^{* *}$ Postal address: Department of Probability and Statistics, University of Sheffield, Sheffield S3 7RH, UK. 
These arise in the solution of the differential equation $\left(d^{k} y / d x^{k}\right)-y=0$; see Kaufman (1955) for a bibliography. There is a known finite form for $J_{k}^{n}(\cdot)$,

$$
J_{k}^{n}(\lambda)=\frac{1}{k} \sum_{j=0}^{k-1} \exp \left(\omega_{k}^{j} \lambda\right) \omega_{k}^{-n j}
$$

Thus we have

$$
\begin{aligned}
\operatorname{Pr}(N \bmod k=n) & =\sum_{m=0}^{\infty} \operatorname{Pr}(N=n+m k) \\
& =\exp (-\lambda) J_{k}^{n}(\lambda) \\
& =\frac{1}{k} \exp (-\lambda) \sum_{j=0}^{k-1} \exp \left(\omega_{k}^{j} \lambda\right) \omega_{k}^{-n j},
\end{aligned}
$$

as required.

Although Lévy mentions the special case $k=2$ of the theorem, in which $J_{2}^{0}(\lambda)=\cosh (\lambda)$ and $J_{2}^{1}(\lambda)=\sinh (\lambda)$, the general connection between the wrapped Poisson distribution and the higher-order hyperbolic functions does not appear to have been made before.

An alternative, probabilistic proof runs as follows. Consider the continuous-time Markov chain $\{X(t) ; t \geqq 0\}$ which cycles through the states $\{0, \cdots, k-1\}$, so that the only permissible transitions are from state $j$ to state $j+1, j=0, \cdots, k-2$, and from state $k-1$ to state 0 . Let all permissible transitions have transition rates equal to $\lambda$, and $X(0)=0$. Since the number of transitions up to time $t$ has the Poisson $(\lambda t)$ distribution, the distribution of $X(1)$ is just the wrapped Poisson distribution on $\{0, \cdots, k-1\}$ with rate $\lambda$.

The generator $Q$ of $\{X(t)\}$ is circulant, so we can readily obtain the eigenvalues of $Q$, which are $\lambda\left(\omega_{k}^{j}-1\right), j=0, \cdots, k-1$, and the corresponding left and right eigenvectors (Bellman (1960), Lancaster (1969)). By standard results (e.g. Karlin and Taylor (1975), p. 152) we can then calculate the probabilities

$$
\operatorname{Pr}(X(1)=n)=\frac{1}{k} \exp (-\lambda) \sum_{j=0}^{k-1} \exp \left(\omega_{k}^{i} \lambda\right) \omega_{k}^{-n j} \quad(n=0,1, \cdots, k-1),
$$

as required.

\section{Acknowledgements}

We are grateful to Dr John Biggins for useful comments on this work. Some of the work was carried out while the second author was supported by an SERC research studentship at the University of Nottingham.

\section{References}

Bellman, R. (1960) Introduction to Matrix Analysis. McGraw-Hill, New York.

Blackwell, P. G. (1990) The Stochastic Modelling of Social and Territorial Behaviour. Unpublished PhD thesis, University of Nottingham.

Karlin, S. and Taylor, H. M. (1975) A First Course in Stochastic Processes, 2nd edn. Academic Press, New York.

Kaufman, H. (1955) A bibliographical note on higher order sine functions. Scripta Math. 28, 29-36.

LANCASTER, P. (1969) Theory of Matrices. Academic Press, New York.

LÉVY, P. (1939) L'addition des variables aléatoires défines sur une circonférence. Bull. Soc. Math. France 67, 1-41.

MARDIA, K. V. (1972) Statistics of Directional Data. Academic Press, New York. 